\title{
Cardiac computed tomography: Beyond the screening of coronary artery disease
}

\section{Tomografía computarizada cardíaca: más allá de la detección de enfermedad coronariân}

\author{
Pablo E. Auquilla Clavijo ${ }^{*}$, Eduardo Pozo Osinalde², and Marina Povar-Echeverría ${ }^{3}$
}

${ }^{1}$ Department of Cardiology, Hospital Royo Villanova, Zaragoza; ${ }^{2}$ Department of Cardiology, Cardiovascular Imaging Unit, Hospital Univerșitario Clínico San Carlos, Madrid; ${ }^{3}$ Department of Internal Medicine, Hospital Universitario Miguel Servet, Zaragoza, Spain

Today, the main use of cardiac computed tomography (CCT) is for the screening of coronary artery disease; however, its use is increasingly common in the follow-up of patients after percutaneous revascularization and in control studies of different clinical scenarios, such as spontaneous coronary artery dissection (SCAD).

SCAD is a major cause of acute coronary syndrome (ACS) in women. According to reports, this entity is the culprit in nearly one-quarter of ACS cases in women younger than 50 years $^{1}$. Although classically it was thought that it affected mostly young women, today it is recognized that it affects women of postmenopausal age as well ${ }^{2}$. In most cases, treatment is usually conservative, but in patients who experience an acute myocardial infarction (AMI) with symptoms of recurrent ischemia or hemodynamic compromise, surgical or percutaneous revascularization may be required ${ }^{3}$. In the case of percutaneous intervention, given the fragility of the vascular wall in these cases, the procedure can have important complications (intramural hematoma and dissection dissemination), secondary to coronary procedures, and/or angioplasty. These complications increase the risk of restenosis or implanted stent fate malposition ${ }^{4}$. For all these reasons, in some cases, it is necessary to carry out control studies to assess implanted stents permeability and/or residual dissection evolution.

We present the case of a 64-year-old woman without cardiovascular risk factors, who had been admitted 3 months prior with an AMI secondary to spontaneous dissection of the anterior descending (AD) artery with total occlusion of the vessel at middle level, complicâted with retrograde peri-procedural dissection toward thelleft common trunk (LCT) and circumflex artery (EX). A drug-eluting stent was implanted in LCT-AD up to the origin of the first diagonal (D1); given that, CX had शे|MI 3 flow, conservative treatment was decided on this yessel. On echocardiography, the left ventricle global systolic
Date of reception: 11-12-2018 50006 Zaragoza, España E-mail: pabloauq50hotmail.com
Available online: 02-20-2019 Arch Cardiol Mex (Eng). 2019;89(3):242-244 www. archivoscardiologia.com
article under the CC BY-NG-ND 2604-7063/@ 2019 Instituto Nacional de Cardiología Ignacio Chávez. Published by Permanyer. This is an open access article under the CC BY-NG-ND 


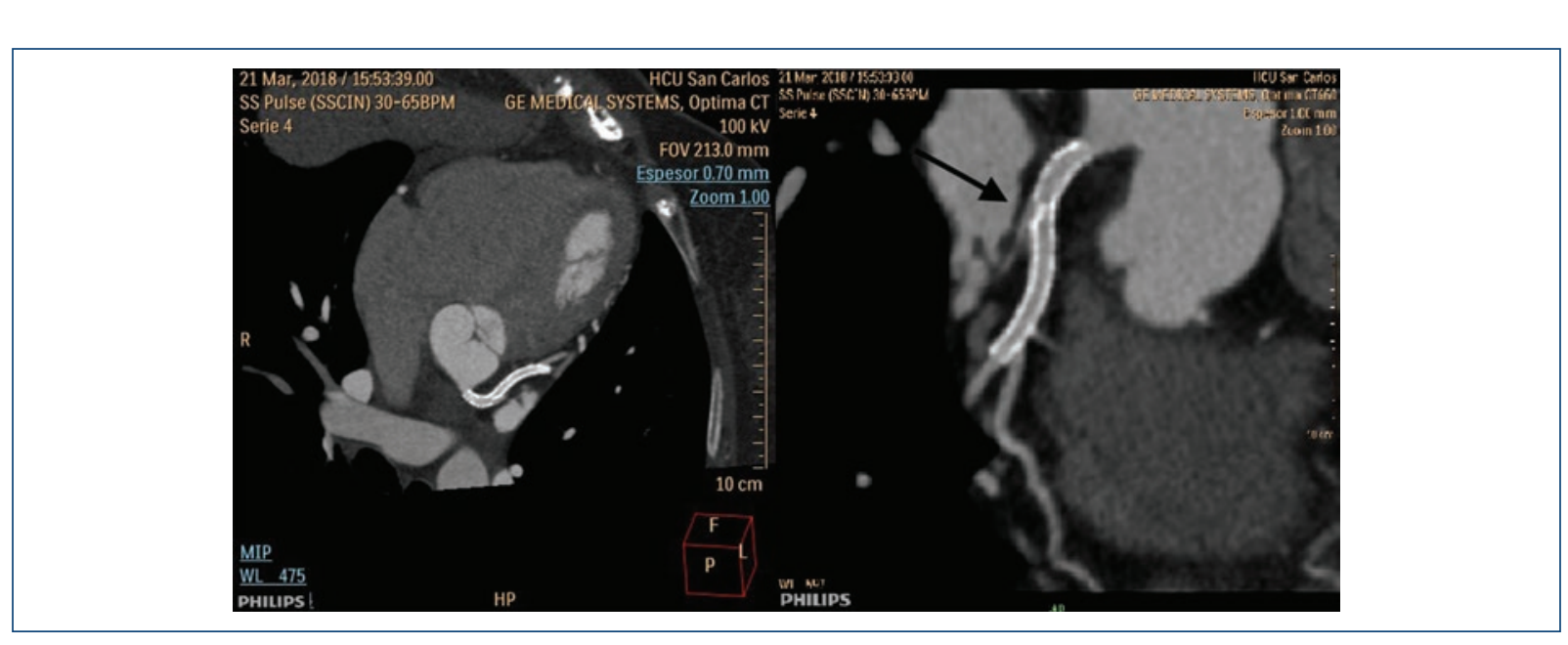

Figure 1. To the right of the image, the presence of a permeable stent from the left common trunk anterior descending artery to the first diagonal origin can be observed in multiplanar reconstruction. In the image on the right, the șâme image is observed in curved planar reconstruction; it can be clearly appreciated how the stent advances up to thefirst diagonal, which is a vessel of good caliber. The arrow shows an image of stent structural alteration performed to cross again toward the circumflex artery; distal to this stent opening, the filling of contrast is normal.

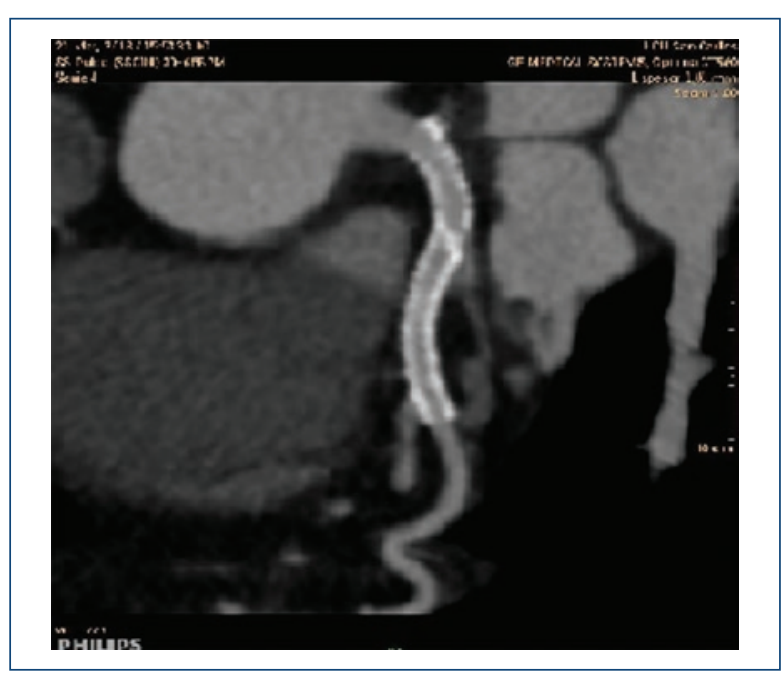

Figure 2. Common left trunk descending artery implanted stent and its advance up to the first diagonal branch, with adequate distal filling thereof, are observed in curved planar reconstruction.

function was found to be preserved, with hypokinesia of the anteroseptal wall middle and apical segments. The patient was discharged asymptomatic with medical treatment. A control CCT was requested at 6 weeks to assess stent permeability and residual dissection.

This study showed the long stent covering the LCT, proximal $A D$ and $D 1$ origin, permeable, and without restenosis, with part of the wall imprinting on AD lumen (Fig. 1). The stent showed structural alteration at the level of CX exit, secondary to the procedure to cross again toward this vessel in the previously performed angioplasty (arrow); distal to this opening, filling of the vessel with contrast is normal (Fig. 2). CX exhibits residual dissection from its exit to the bifurcation withethe first obtuse marginal, with adequate filling with contrast in the distal vessel (Fig. 3). The study shows that all implanted stents are permeable and an absence of residual dissection progression in CX. The patient remained asymptomatic throughout the follow-up.

Although in most cases the diagnostic technique of choice to assess stent permeability is invasive coronary angiography, there are series showing that, in stênts implanted in the LCT and epicardial vessels proximal segments, CCT can offer high-quality images to assess implanted stents' permeability ${ }^{5}$. In our case, the high usefulness of CCT as a non-invasive test in the assessment of stent structural integrity, as well as the excellent anatomical definition of vascular wall pathologies, as in this case, of a coronary dissection, is shown.

\section{Conflicts of interest}

The authors declare that they have no conflicts of interest.

\section{Funding}

No sponsorship of any kind was received to carryout this article. 


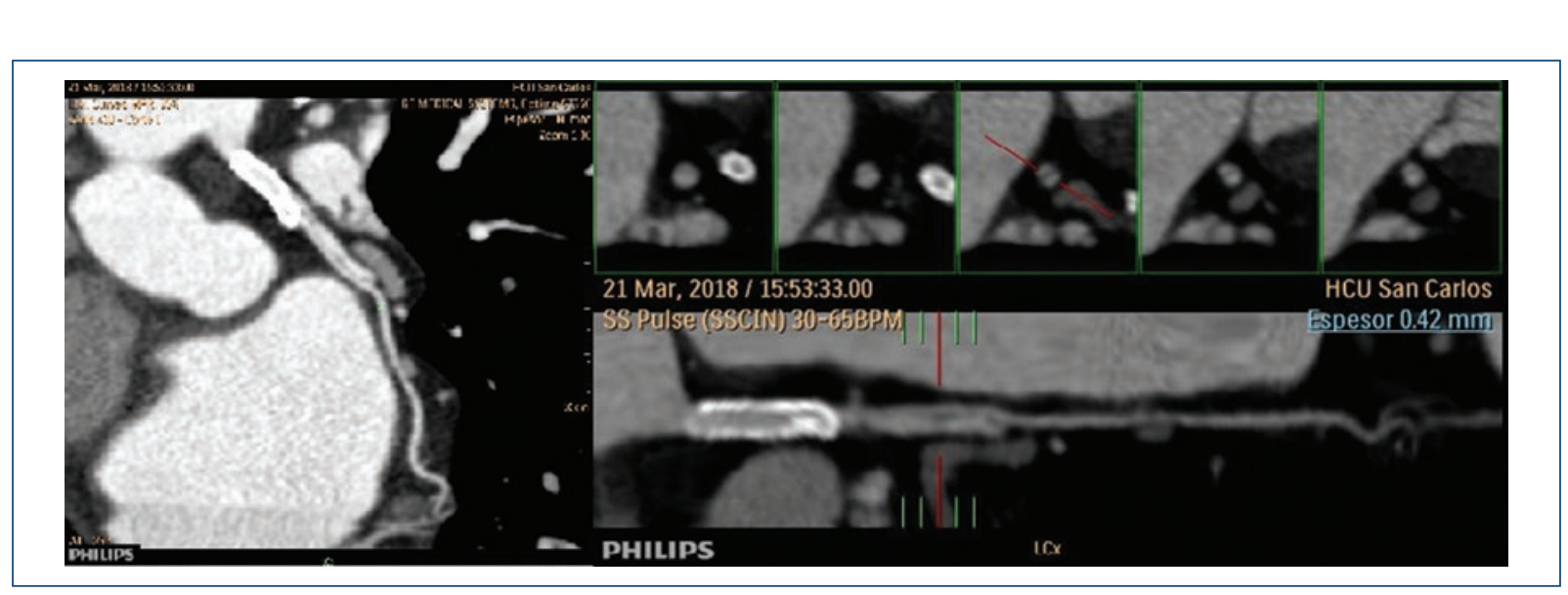

Figure 3. Image of the circumflex artery dissection from its origin to the bifurcation with the first obtuse marginal branch is observed in curved planar reconstruction. Appropriate contrast filling in the distal vessel is appreciated.

\section{Ethical disclosures}

Protection of people and animal subjects. The authors declare that no experiments were performed on humans or animals for this study.

Confidentiality of data. The authors declare that they have followed the protocols of their work center on the publication of patient data.

Right to privacy and informed consent. The authors have obtained written informed consent of the patients and/or subjects mentioned in the article. The corresponding author is in possession of this document.

\section{References}

1. Saw J, Aymong E, Mancini GB, Sedlak T, Starovoytov A, Ricei D. Nonatherosclerotic coronary artery disease in young women. Can JCardiol. 2014;30:814-9.

2. Saw J, Humphries K, Aymong E, Sedlak T, Prakash R, Starovoytov A, et al. Spontaneous coronary artery dissection: clinical outcomes and risk of recurrence. J Am Coll Cardiol. 2017;70:1148-58.

3. Hayes SN, Kim ESH, Saw J, Adlam D, Arslanian-Engoren C, Economy KE, et al. Spontaneous coronary artery dissection: current state of the science: A scientific statement from the American Heart Association. Circulation. 2018;137:e523-57.

4. Tweet MS, Hayes SN, Pitta SR, Simari RD, Lerman A, Lennon RJ et al. Clinical features, management, and prognosis of spontaneous coronary artery dissection. Circulation. 2012;126(5):579-88.

5. Ehara M, Kawai M, Surmely JF, Matsubara T, Terashima M, Tsuchikane E, et al. Diagnostic accuracy of coronary in-stent restenosis using 64-slice computed tomography: comparison with invasive coronary anglography. J Am Coll Cardiol. 2007;49:951-9. 